\title{
Fatores associados ao enfrentamento da pandemia da COVID-19 por pessoas idosas com comorbidades
}

\author{
Factors associated with coping with the COVID-19 pandemic by older adults with comorbidities \\ Factores asociados al afrontamiento de la pandemia COVID-19 por adultos mayores con \\ comorbilidades
}

\author{
Zilmar Augusto de Souza Filho ${ }^{1}$ (i) \\ Camila Rodrigues Barbosa Nemer ${ }^{2}$ (b) \\ Elizabeth Teixeira ${ }^{3}$ (1) \\ André Luiz Machado das Neves $^{3}$ (D) \\ Marcia Helena Machado Nascimento 4 (1) \\ Horacio Pires Medeiros 5 (1) \\ Bruna Alessandra Costa e Silva Panarra ${ }^{6,7}$ (1) \\ Paula Andreza Viana Lima ${ }^{1}$ (D) \\ Vanessa Calmont Gusmão Gigante ${ }^{8}$ (1) \\ Vera Lúcia Gomes de Oliveira ${ }^{4}$ (])
}

\section{Universidade Federal do Amazonas.}

Manaus, AM, Brasil.

2. Universidade Federal do Amapá. Macapá, AP, Brasil.

3. Universidade do Estado do Amazonas. Manaus, AM, Brasil.

4. Universidade do Estado do Pará. Belém, PA, Brasil.

5. Universidade Estácio. Castanhal, PA, Brasil.

6. Universidade Maurício de Nassau. Belém, Pará, Brasil.

7. Universidade da Amazônia. Belém, PA, Brasil.

8. Universidade Federal do Mato Grosso do Sul. Campo Grande, MS. Brasil.

Autor correspondente:

Elizabeth Teixeira.

E-mail: etfelipe@hotmail.com.

Recebido em 17/12/2020.

Aprovado em 19/03/2021.

DOI:https://doi.org/10.1590/2177-9465-EAN-2020-0495

\begin{abstract}
Resumo
Objetivo: identificar fatores associados ao enfrentamento da pandemia da COVID-19 por pessoas idosas com e sem comorbidades. Método: estudo descritivo, transversal, com pessoas idosas $(n=569)$, entre 60 e 80 anos, com ou sem comorbidades, nas cinco regiões do Brasil. Coleta de dados com questionário virtual e análise com base na estatística descritiva e inferencial. Resultados: os resultados mostram que $351,(61,68 \%)$, referem comorbidade. Houve associação significativa entre os grupos nas variáveis: faixa etária $(p=0,017)$, realizar alguma atividade laboral $(p \leq 0,001)$, pensamento da possibilidade de ser infectado pelo novo coronavírus ( $p \leq 0,001)$, concordar com medidas de prevenção adotadas para o distanciamento social $(p \leq 0,001)$, se informar por outro meio de comunicação além da televisão $(p \leq 0,001)$. Conclusão e implicações para a prática: os idosos com comorbidades pensam na possibilidade de ser infectado pelo novo coronavírus, concordam mais com as medidas de distanciamento social e se informam mais. Nesse sentido, indica-se a realização de pesquisas com ênfase nos idosos sem comorbidade, para direcionar melhor os cuidados de saúde em tempos de pandemias.
\end{abstract}

Palavras-chave: Idoso; Pandemias; Infecções por coronavírus; Estudos transversais; Distanciamento social.

\section{Abstract}

Objective: to identify factors associated to coping with the COVID-19 pandemic by older adults with and without comorbidities Method: a descriptive, cross-sectional study with older adults $(n=569)$, aged between 60 and 80 years old, with or without comorbidities, in the five Brazilian regions. Data collection with virtual questionnaire and analysis based on descriptive and inferential statistics. Results: the results show that $351(61.68 \%)$ refer to comorbidity. There was a significant association between the groups in the following variables: age group $(p=0.017)$, performing some work activity $(p \leq 0.001)$, thinking about the possibility of being infected by the new coronavirus $(p \leq 0.001)$, agreeing with prevention measures adopted for social distancing ( $p \leq 0.001)$, informing yourself by other means of communication besides television $(p \leq 0.001)$. Conclusion and implications for the practice: the older adults with comorbidities think about the possibility of being infected by the new coronavirus, agree more with the social distancing measures and get more information. To such an effect, it is recommended to carry out research studies with an emphasis on the older adult without comorbidity, to better target health care in pandemic times.

Keywords: Aged; Pandemics; Coronavirus infections; Cross-sectional; Social distancing.

\section{RESUMEN}

Objetivo: identificar factores asociados al afrontamiento de la pandemia COVID-19 en adultos mayores con y sin comorbilidades Método: estudio descriptivo, transversal con personas mayores $(n=569)$, entre 60 y 80 años, con o sin comorbilidades, en las cinco regiones de Brasil. La recolección de datos se realizó con cuestionario virtual y el análisis mediante estadística descriptiva e inferencial. Resultados: los resultados muestran que 351 personas, $(61,68 \%)$, refieren a comorbilidades. Hubo asociación significativa entre grupos en las variables: grupo de edad $(p=0,017)$, realizar alguna actividad laboral $(p \leq 0,001)$, pensar en la posibilidad de estar infectado por el nuevo coronavirus $(p \leq 0,001)$, acuerdo con las medidas preventivas adoptadas para e distanciamiento social ( $\mathrm{p} \leq 0.001$ ), informarse por otros medios de comunicación además de la televisión ( $p \leq 0.001)$. Conclusión e implicaciones para la práctica: los adultos mayores con comorbilidades piensan en la posibilidad de estar contagiados por el nuevo coronavirus, están más de acuerdo con las medidas de distanciamiento social y obtienen más información. En este sentido, se recomienda realizar una investigación con énfasis en los adultos mayores sin comorbilidades, para orientar mejor la atención sanitaria en tiempos de pandemia.

Palabras clave: Adulto mayor; Pandemias; Infecciones por coronavírus; Estudios transversales. distanciamiento social. 


\section{INTRODUÇÃO}

Uma nova infecção viral foi descrita pela primeira vez na cidade de Wuhan, na província de Hubei, China. ${ }^{1} \mathrm{O}$ agente, até então desconhecido, foi identificado como novo coronavírus, causador da doença nominada COVID-19. A Organização Mundial da Saúde, preocupada com a difusão global do surto, e sua magnitude, anunciou a pandemia da COVID-19, em 11 de março de 2020 . $^{2}$

A realização de estudos rapidamente deflagrados em inúmeros países por conta da condição de emergência, possibilitou constatar a prevalência de comorbidades nos pacientes com infecções por coronavírus, com destaque para hipertensão, diabetes, doenças respiratórias e cardiovasculares. As comorbidades citadas, foram correlacionadas como fatores de risco para pacientes graves internados pela COVID-19, em comparação com pacientes não graves. ${ }^{3,4}$

Nesse contexto, verificou-se que pessoas idosas têm maior probabilidade de evoluir para um estado grave, bem como de manifestarem as comorbidades supracitadas e, consequentemente, uma taxa elevada expressa em 5,56\% de mortalidade relacionada à infecção por coronavírus, quando comparado ao grupo de pacientes jovens e de meia idade, que foi de 5,26\%. ${ }^{4}$

Um estudo nos Estados Unidos, sobre mortes provocadas pela COVID-19, apontou 39 mortes em crianças de um ano; 79 mortes entre 1 a 14 anos; 525 mortes entre 15 a 24 anos; 2.278 mortes entre 25 a 34 anos; 5.991 mortes entre 35 a 44 anos; 16.282 mortes entre 45 a 54 anos; 40.758 mortes entre 55 a 64 anos; 73.856 mortes entre 65 a 74 anos; 95.848 mortes entre 75 a 84 anos e 111.475 mortes acima de 85 anos. $^{5}$

As autoridades internacionais de saúde, de vários países, estão alertando que as pessoas idosas correm risco de complicações mais graves e possível letalidade no caso de infecção pelo novo coronavírus. Destaca-se que o risco de doença grave pela COVID-19 e seu desdobramento em morte, pode agravar com a idade. ${ }^{6}$

No Brasil, até o final da semana epidemiológica 52 de 2020 , no dia 26 de dezembro, foram confirmados 7.465 .806 casos de infecções por coronavírus, o terceiro país com o maior número de casos acumulados, ficando atrás da Índia (10.187.850) e dos Estados Unidos (18.982.634). Em relação aos óbitos acumulados, foram confirmados 190.795 no Brasil, ficando atrás somente dos Estados Unidos (331.909). ${ }^{7}$

Tal quadro é agravado quando emergem controvérsias sobre as políticas de prevenção, pondo em debate a questão das normas de distanciamento social e, muitas vezes, levantando dúvidas sobre qual diretriz adotar, o que pode levar a falhas e prejuízos nos modos de prevenção, que estão sendo adotados pelos gestores das cidades; disseminação de informações falsas sobre tratamento, prevenção e modos de contágio; e medidas não comprovadas cientificamente tão presentes na pandemia do novo coronavírus ${ }^{8}$. Esses agravamentos não contribuem em nada para o enfrentamento coletivo e racional da situação em tela.

A pandemia da COVID-19 tem afetado a vida cotidiana das pessoas levando a uma desorganização, que só se experimenta em meio aos levantes e às revoluções, o que faz com que se repense toda a estrutura de vida atual. ${ }^{9}$

As bases da justificativa da investigação realizada são as mudanças de comportamento de pessoas idosas no enfrentamento da pandemia, as reações emocionais, além da identificação de como pensam e se manifestam sobre as medidas adotadas nas cidades para o distanciamento social. As pessoas idosas são identificadas como "grupo de risco", e as principais medidas são indicadas exatamente à essa população.

Nessa perspectiva, o presente estudo objetivou identificar fatores associados ao enfrentamento da pandemia da COVID-19 por pessoas idosas com e sem comorbidade.

\section{MÉTODO}

Trata-se de um estudo descritivo, do tipo estudo transversal, realizado com pessoas idosas nas cinco regiões do Brasil. A pesquisa ocorreu entre os meses de abril a junho de 2020. Foram analisados dados de pessoas com idade igual ou superior a 60 anos, a partir do envio de um formulário eletrônico, inserido no Google Forms, disponibilizado por e-mail e/ou whatsapp.

Em decorrência do contexto da pandemia da COVID-19 e das recomendações do distanciamento social, o pré-teste do instrumento elaborado para a pesquisa foi realizado apenas pela equipe e profissionais com expertise em saúde do idoso que já realizaram estudos em parceria com os autores.

A composição da amostra dos sujeitos deste estudo foi apoiada na técnica de snowball sampling, por amostragem não probabilística, o que permitiu que os participantes iniciais indicassem os novos sujeitos e assim sucessivamente. A amostra final foi composta por 569 pessoas idosas, que aceitaram voluntariamente participar da pesquisa, mediante aceite do Termo de Consentimento Livre e Esclarecido (TCLE).

Em relação à variável dependente, os fatores associados foram analisados a partir da presença ou não de diagnóstico clínico de comorbidades crônicas autorreferidas pelos participantes, consideradas de risco para a COVID-19, tais como: hipertensão arterial, diabetes mellitus, neoplasias, asma, hipotireoidismo, além de outras doenças pulmonares, cardiovasculares e osteoarticulares.

As variáveis independentes foram de caracterização socioeconômica como: sexo, faixa-etária, região onde reside e atividade ocupacional. Além de 14 variáveis subjetivas não diretamente observáveis que descreveram as emoções das pessoas idosas relacionadas ao medo da COVID-19, como tristeza, pânico, choro e o pensamento sobre a possibilidade de vir a ser infectado pelo novo coronavírus; à adoção de medidas de prevenção: cumprimento das orientações de distanciamento social, concordância com as medidas de distanciamento, percepção do cumprimento das medidas de distanciamento social pela população, adesão às medidas estabelecidas na cidade que reside; e ao recebimento de informações veiculadas a respeito da COVID-19: surgimento de dúvidas das informações que circulavam, amenização de dúvidas com outras pessoas, acompanhamento de informações pela televisão ou outro meio de 
comunicação, e a satisfação com as informações veiculadas. Para a mensuração dessas variáveis utilizou-se a escala do tipo likert.

Para o tratamento dos dados obtidos, organizou-se um banco de dados em uma planilha do programa Excel $($, Microsoft Office 365 , e analisados no software Stata versão 13,0 . As variáveis categóricas foram descritas por frequências absolutas e relativas. Os dados foram tratados segundo o teste bivariado do Qui-quadrado de Pearson e para verificar a diferença estatística entre os desfechos adotou-se o valor de $p \leq 0,05$.

Para verificar a associação entre as variáveis dependentes e as variáveis independentes do estudo foram estimadas as razões de chance por Odds Ratio (OR) bruto a partir do modelo de regressão logística binária com respectivos intervalos de confiança de 95\% (IC95\%). Para a seleção das variáveis, estabeleceu-se um nível de significância de $20 \%(p \leq 0,20)$. O ajuste global do modelo foi verificado através do teste da razão de verossimilhanças.

O estudo foi aprovado pelo Comitê de Ética em Pesquisa da Universidade Federal do Amapá, ao atender as recomendações da Resolução do Conselho Nacional de Saúde nำ 466/12, Parecer no. 4.061.643.

\section{RESULTADOS}

Responderam ao formulário 569 pessoas idosas (Tabela 1), destas, 351 (61,68\%) afirmaram apresentar algum tipo de comorbidade. No que tange ao sexo das pessoas idosas, destacase que $72,26 \%$ ( $n=414$ ) eram do sexo feminino. Aponta-se que cerca $75 \%$ das pessoas idosas na faixa etária entre 75 a 79 anos e faixa etária 80 ou mais anos, apresentaram comorbidades.

Tabela 1. Caracterização do perfil sociodemográfico das pessoas idosas, Brasil, 2020.

\begin{tabular}{|c|c|c|c|c|}
\hline Variáveis & $\begin{array}{c}\text { Com comorbidade } \\
n=351\end{array}$ & $\begin{array}{c}\text { Sem comorbidade } \\
n=\mathbf{2 1 8}\end{array}$ & Total $n=569$ & Valor $\mathrm{p}^{*}$ \\
\hline & n (\%) & n (\%) & n (\%) & \\
\hline Sexo & & & & 0,277 \\
\hline Masculino & $90(58,06)$ & $65(41,94)$ & $155(27,24)$ & \\
\hline Feminino & $261(63,04)$ & $153(36,96)$ & $414(72,76)$ & \\
\hline Faixa etária & & & & 0,017 \\
\hline 60-64 anos & $142(55,04)$ & $116(44,96)$ & $258(45,34)$ & \\
\hline $65-69$ anos & $102(64,56)$ & $56(35,44)$ & $158(27,77)$ & \\
\hline 70-74 anos & $50(64,10)$ & $28(35,90)$ & $78(13,71)$ & \\
\hline 75-79 anos & $24(75,00)$ & $8(25,00)$ & $32(5,62)$ & \\
\hline$\geq 80$ anos & $33(76,74)$ & $10(23,26)$ & $43(7,56)$ & \\
\hline Região da residência & & & & 0,813 \\
\hline Norte & $91(59,48)$ & $62(40,52)$ & $153(26,89)$ & \\
\hline Nordeste & $29(61,70)$ & $18(38,30)$ & $47(8,26)$ & \\
\hline Centro-oeste & $23(62,16)$ & $14(37,84)$ & $37(6,50)$ & \\
\hline Sudeste & $186(63,70)$ & $106(36,30)$ & $292(51,32)$ & \\
\hline Sul & $22(55,00)$ & $18(45,00)$ & $40(7,03)$ & \\
\hline Atividades & & & & $\leq 0,001$ \\
\hline Sem atividade & $202(64,33)$ & $112(35,67)$ & $314(55,18)$ & \\
\hline Professor & $45(60,81)$ & $29(39,19)$ & $74(13,01)$ & \\
\hline Do lar & $35(77,78)$ & $10(22,22)$ & $45(7,91)$ & \\
\hline Servidor público & $12(66,67)$ & $6(33,33)$ & $18(3,16)$ & \\
\hline Profissional de saúde & $20(74,07)$ & $7(25,93)$ & $27(4,75)$ & \\
\hline $\begin{array}{l}\text { Aposentado, } \\
\text { exercendo atividade }\end{array}$ & $1(33,33)$ & $2(66,67)$ & $3(0,53)$ & \\
\hline Outras atividades & $36(40,91)$ & $52(59,09)$ & $88(15,47)$ & \\
\hline
\end{tabular}

*Teste Qui-quadrado de Pearson. FONTE: dados da pesquisa, 2020 
Quanto a exercer atividade laboral, destaca-se que $77,78 \%$ das pessoas que declararam serem do lar possuíam alguma comorbidade, assim como $74,07 \%$ dos profissionais de saúde. Houve diferenças estatisticamente significantes entre os grupos nas respectivas variáveis: faixa etária $(p=0,017)$ e realizar alguma atividade laboral $(p \leq 0,001)$.

A Tabela 2 apresenta as características e os fatores associados às emoções sentidas pelas pessoas idosas relacionadas a
COVID 19, medidas de prevenção e informações veiculadas para o enfrentamento da pessoa idosa ao novo coronavírus.

De acordo com as informações apresentadas na Tabela 2, os grupos apresentaram diferenças estatisticamente significantes em relação às variáveis: ao pensamento da possibilidade de vir a ser infectado pelo novo coronavírus $(p \leq 0,001)$, concordar com medidas de prevenção adotadas para o distanciamento social $(p \leq 0,001)$ e se informar por outro meio de comunicação

Tabela 2. Caracterização e fatores associados às emoções, medidas de prevenção e informações veiculadas para o enfrentamento da pessoa idosa ao novo coronavírus, Brasil, 2020.

\begin{tabular}{|c|c|c|c|c|c|}
\hline \multirow{2}{*}{ Variáveis } & Com comorbidade & Sem comorbidade & \multirow{2}{*}{ Valor $\mathrm{p}^{*}$} & \multirow{2}{*}{ OR [IC95\%] } & \multirow{2}{*}{ Valor $p$} \\
\hline & n (\%) & n (\%) & & & \\
\hline \multicolumn{2}{|c|}{ Sentir medo da COVID } & & 0,060 & & \\
\hline Nenhuma vez & $71(65,74)$ & $37(34,26)$ & & 1 & \\
\hline Algumas vezes & $130(55,08)$ & $106(44,92)$ & & $0,63[0,39-1,02]$ & 0,064 \\
\hline Indiferente & $17(58,62)$ & $12(41,38)$ & & $0,73[0,31-1,70]$ & 0,478 \\
\hline Muitas vezes & $87(65,91)$ & $45(34,09)$ & & $1,00[0,58-1,72]$ & 0,978 \\
\hline Sempre & $46(71,88)$ & $18(28,13)$ & & $1,33[0,67-2,61]$ & 0,405 \\
\hline \multicolumn{2}{|l|}{ Ter tristeza } & & 0,586 & & \\
\hline Nenhuma vez & $87(64,93)$ & $47(35,07)$ & & 1 & \\
\hline Algumas vezes & $146(58,63)$ & $103(41,37)$ & & $0,76[0,49-1,18]$ & 0,230 \\
\hline Indiferente & $12(60,00)$ & $8(40,00)$ & & $0,81[0,30-2,12]$ & 0,668 \\
\hline Muitas vezes & $83(65,87)$ & $43(34,13)$ & & $1,04[0,62-1,73]$ & 0,872 \\
\hline Sempre & $23(57,50)$ & $17(42,50)$ & & $0,73[0,35-1,50]$ & 0,394 \\
\hline \multicolumn{2}{|l|}{ Sentir Pânico } & & 0,212 & & \\
\hline Nenhuma vez & $225(61,48)$ & $141(38,52)$ & & 1 & \\
\hline Algumas vezes & $85(60,28$ & $56(39,72)$ & & $0,95[0,63-1,41]$ & 0,805 \\
\hline Indiferente & $10(47,62)$ & $11(52,38)$ & & $0,56[0,23-1,37]$ & 0,211 \\
\hline Muitas vezes & $28(77,78)$ & $8(22,22)$ & & $2,19[0,97-4,94]$ & 0,058 \\
\hline Sempre & $3(60,00)$ & $2(40,00)$ & & $0,94[0,15-5,69]$ & 0,946 \\
\hline \multicolumn{2}{|l|}{ Ter chorado } & & 0,152 & & \\
\hline Nenhuma vez & $204(60,71)$ & $132(39,29)$ & & 1 & \\
\hline Algumas vezes & $92(57,86)$ & $67(42,14)$ & & $0,88[0,60-1,30]$ & 0,546 \\
\hline Indiferente & $12(66,67)$ & $6(33,33)$ & & $1,29[0,47-3,53]$ & 0,615 \\
\hline Muitas vezes & $33(76,74)$ & $10(23,26)$ & & $2,13[1,01-4,47]$ & 0,045 \\
\hline Sempre & $10(76,92)$ & $3(23,08)$ & & $2,15[0,58-7,98]$ & 0,250 \\
\hline \multicolumn{3}{|c|}{$\begin{array}{l}\text { Pensamento da possibilidade de vir a ser infectado pelo novo } \\
\text { coronavírus }\end{array}$} & $\leq 0,001$ & & \\
\hline Nenhuma vez & $142(54,83)$ & $117(45,17)$ & & 1 & \\
\hline Algumas vezes & $124(61,69)$ & $77(38,31)$ & & $1,32[0,91-1,93]$ & 0,140 \\
\hline Indiferente & $28(71,79)$ & $11(28,21)$ & & $2,09[1,00-4,39]$ & 0,050 \\
\hline Muitas vezes & $24(70,59)$ & $10(29,41)$ & & $1,97[0,90-4,30]$ & 0,086 \\
\hline Sempre & $33(91,67)$ & $3(8,33)$ & & $9,06[2,71-30,30]$ & $\leq 0,001$ \\
\hline
\end{tabular}

*Teste Qui-quadrado de Pearson. FONTE: dados da pesquisa, 2020 
Tabela 2. Continuação...

\begin{tabular}{|c|c|c|c|c|c|}
\hline \multirow{2}{*}{ Variáveis } & \multirow{2}{*}{$\frac{\text { Com comorbidade }}{\mathrm{n}(\%)}$} & \multirow{2}{*}{$\frac{\text { Sem comorbidade }}{\mathrm{n}(\%)}$} & \multirow{2}{*}{ Valor $p^{*}$} & \multirow{2}{*}{ OR [IC95\%] } & \multirow{2}{*}{ Valor $\mathrm{p}$} \\
\hline & & & & & \\
\hline \multicolumn{3}{|c|}{ Seguir o distanciamento social } & 0,200 & & \\
\hline Nenhuma vez & $1(33,33)$ & $2(66,67)$ & & 1 & \\
\hline Algumas vezes & $23(63,89)$ & $13(36,11)$ & & $3,53[0,29-42,88]$ & 0,321 \\
\hline Muitas vezes & $59(52,68)$ & $53(47,32)$ & & $2,22[0,19-25,26]$ & 0,518 \\
\hline Sempre & $266(64,10)$ & $149(35,90)$ & & $3,57[0,32-39,70]$ & 0,300 \\
\hline \multicolumn{3}{|c|}{ Concordar com medidas de distanciamento social } & $\leq 0,001$ & & \\
\hline Nenhuma vez & $7(36,84)$ & $12(63,16)$ & & 1 & \\
\hline Sempre & $190(68,84)$ & $86(31,16)$ & & $3,78[1,44-9,95]$ & 0,007 \\
\hline \multicolumn{3}{|c|}{$\begin{array}{l}\text { Perceber o cumprimento das medidas de distanciamento social } \\
\text { pela população }\end{array}$} & 0,189 & & \\
\hline Nenhuma vez & $14(56,00)$ & $11(44,00)$ & & 1 & \\
\hline Algumas vezes & $210(63,83)$ & $119(36,17)$ & & $1,38[0,61-3,15]$ & 0,435 \\
\hline Indiferente & $11(78,57)$ & $3(21,43)$ & & $2,88[0,64-12,92]$ & 0,167 \\
\hline Muitas vezes & $72(54,14)$ & $61(45,86)$ & & $0,92[0,39-2,19]$ & 0,864 \\
\hline Sempre & $44(64,71)$ & $24(35,29)$ & & $1,44[0,56-3,66]$ & 0,443 \\
\hline Sempre & $262(65,01)$ & $141(34,99)$ & & $1,85[0,37-9,32]$ & 0,452 \\
\hline \multicolumn{3}{|c|}{ Sentir dúvidas das informações que circulavam } & 0,629 & & \\
\hline Nenhuma vez & $105(63,25)$ & $61(36,75)$ & & 1 & \\
\hline Algumas vezes & $159(56,11)$ & $110(40,89)$ & & $0,83[0,56-1,25]$ & 0,390 \\
\hline Indiferente & $5(50,00)$ & $5(50,00)$ & & $0,58[0,16-2,08]$ & 0,405 \\
\hline Muitas vezes & $64(65,66)$ & $34(34,34)$ & & $1,11[0,65-1,87]$ & 0,693 \\
\hline Sempre & $17(68,00)$ & $8(32,00)$ & & $1,23[0,50-3,02]$ & 0,645 \\
\hline \multicolumn{3}{|c|}{ Tirar as dúvidas com outras pessoas } & 0,281 & & \\
\hline Nenhuma vez & $61(62,89)$ & $36(37,11)$ & & 1 & \\
\hline Algumas vezes & $130(60,19)$ & $86(39,81)$ & & $0,89[0,54-1,46]$ & 0,650 \\
\hline Indiferente & $5(35,71)$ & $9(64,29)$ & & $0,32[0,10-1,05]$ & 0,061 \\
\hline Muitas vezes & $61(62,24)$ & $37(37,76)$ & & $0,97[0,54-1,73]$ & 0,926 \\
\hline Sempre & $94(65,28)$ & $50(34,72)$ & & $1,10[0,64-1,89]$ & 0,704 \\
\hline
\end{tabular}

*Teste Qui-quadrado de Pearson. FONTE: dados da pesquisa, 2020 
Tabela 2. Continuação...

\begin{tabular}{|c|c|c|c|c|c|}
\hline \multirow{2}{*}{ Variáveis } & Com comorbidade & Sem comorbidade & \multirow{2}{*}{ Valor $p^{*}$} & \multirow{2}{*}{ OR [IC95\%] } & \multirow{2}{*}{ Valor $p$} \\
\hline & $\mathrm{n}(\%)$ & $\mathrm{n}(\%)$ & & & \\
\hline \multicolumn{3}{|c|}{ Acompanhar informações pela TV } & 0,060 & & \\
\hline Nenhuma vez & $9(39,13)$ & $14(60,87)$ & & 1 & \\
\hline Algumas vezes & $88(61,11)$ & $56(38,89)$ & & $2,44[0,99-6,02]$ & 0,052 \\
\hline Indiferente & $5(55,56)$ & $4(44,44)$ & & $1,94[0,40-9,24]$ & 0,403 \\
\hline Muitas vezes & $69(56,56)$ & $53(43,44)$ & & $2,02[0,81-5,03]$ & 0,129 \\
\hline Sempre & $180(66,42)$ & $91(33,58)$ & & $3,07[1,28-7,37]$ & 0,012 \\
\hline \multicolumn{3}{|c|}{ Se informar por outro meio de comunicação além da TV } & $\leq 0,001$ & & \\
\hline Nenhuma vez & $81(78,64)$ & $22(21,36)$ & & 1 & \\
\hline Algumas vezes & $107(58,79)$ & $75(41,21)$ & & $0,38[0,22-0,67]$ & $\leq 0,001$ \\
\hline Indiferente & $6(60,00)$ & $4(40,00)$ & & $0,40[0,10-1,57]$ & 0,192 \\
\hline Muitas vezes & $78(53,06)$ & $69(46,94)$ & & $0,30[0,17-0,54]$ & $\leq 0,001$ \\
\hline Sempre & $79(62,20)$ & $48(37,80)$ & & $0,44[0,24-0,80]$ & 0,008 \\
\hline \multicolumn{3}{|c|}{ Estar satisfeito com as informações veiculadas } & 0,130 & & \\
\hline Nenhuma vez & $19(43,18)$ & $25(56,82)$ & & 1 & \\
\hline Algumas vezes & $156(62,40)$ & $94(37,60)$ & & $2,18[1,14-4,17]$ & 0,018 \\
\hline Indiferente & $11(61,11)$ & $7(38,89)$ & & $2,06[0,67-6,33]$ & 0,204 \\
\hline Muitas vezes & $73(64,04)$ & $41(35,96)$ & & $2,34[1,15-4,75]$ & 0,019 \\
\hline Sempre & $92(64,34)$ & $51(35,66)$ & & $2,37[1,19-4,72]$ & 0,014 \\
\hline
\end{tabular}

*Teste Qui-quadrado de Pearson. FONTE: dados da pesquisa, 2020

além da TV $(p \leq 0,001)$. Não houve diferenças significativas nas demais variáveis.

Evidenciou-se a associação positiva nas emoções das pessoas idosas com comorbidades quanto a possuir duas vezes maior chance de ter chorado muitas vezes durante a pandemia (OR=2,13 IC95\% 1,01-4,47).

Em relação às medidas de prevenção, observou-se diferenças entre os grupos estudados apontando que as pessoas idosas com comorbidades tem três vezes maior chance (OR=3,78 IC95\% $1,44-9,95)$ de concordar com as medidas de distanciamento social ou concordam muitas vezes (OR=2,79 IC95\% 1,03-7,59) com essas medidas.

Quanto às ações de enfrentamento relacionadas a aquisição de informações sobre a COVID-19, observou-se que as pessoas idosas com comorbidades possuem três vezes maior chance (OR=3,07 IC95\% 1,28-7,37) de sempre acompanhar as informações transmitidas pela televisão. E ainda possuem a maior chance de estarem sempre (OR=2,37 IC95\% 1,19-4,72) e muitas vezes OR=2,34 IC95\% 1,15-4,75) satisfeitos ou até mesmo algumas vezes satisfeitos (OR=2, $18 \mathrm{IC} 95 \% 1,14-4,17)$ com as informações que estão sendo veiculadas a respeito do novo coronavírus.

Merece destaque entre os resultados obtidos, que a utilização de outros meios de comunicação para aquisição de informações revelou-se como fator de proteção entre as pessoas idosas com comorbidades.

\section{DISCUSSÃO}

A presença de comorbidade entre as pessoas idosas, observada neste estudo $(61,68 \%)$ foi menor à observada em pesquisas realizadas no Ceará $(75 \%)^{10}$ e Florianópolis $(93,2 \%) .{ }^{11}$ No contexto da pandemia do novo coronavírus, as comorbidades já existentes se apresentam como um fator de risco para populações acima de 60 anos. Em estudo realizado no Maranhão, ${ }^{12}$ evidenciou-se que nos óbitos ocorridos pela COVID-19, a faixa etária predominante foi a de 60 anos ou mais e dentre as comorbidades relacionadas aos óbitos registrados por infecções por coronavírus, houve maior ocorrência de doenças crônicas do sistema cardiovascular e imunológico.

Estudo realizado na China ${ }^{13}$, que avaliou 150 casos de infecção laboratorialmente confirmada por SARS-CoV-2, evidenciou que pacientes com doença cardiovascular associada à COVID-19 tiveram maior risco de morte, além de que houve diferença estatisticamente significante para idade avançada nos pacientes que evoluíram a óbito $(p<0,001)$.

Estudos internacionais ${ }^{14,15}$ afirmam que os idosos são mais suscetíveis aos efeitos psicológicos em uma pandemia, podendo 
manifestar emoções como tristeza, ansiedade, estresse, raiva, medo e choro. Os resultados desta pesquisa também revelaram que pessoas idosas com comorbidades possuíam duas vezes maior chance de ter chorado muitas vezes durante a pandemia, quando comparado aos sem comorbidades, o que pode ser explicado pela incerteza da cura e/ou da morte do idoso com o percurso clínico da doença caso seja infectado.

Estudo realizado com idosos com comorbidades/doenças crônicas antes da pandemia evidenciou que só o fato de ter uma doença crônica trazia sentimentos negativos e sofrimento emocional para os idosos participantes do estudo, ${ }^{16}$ sugerese, portanto, que com a pandemia isso possa ter se agravado. Ademais, o choro pode ser uma manifestação de transtornos mentais, como pode ser observado em estudo realizado com idosos chineses, o qual, evidenciou que $37,1 \%$ dos participantes experimentaram depressão e ansiedade durante a pandemia da COVID-19. ${ }^{17}$

Para entender a fragilidade no idoso, faz-se necessário ter atenção não só com as alterações físicas e fisiológicas em decorrência do processo de envelhecimento, mas também para possíveis alterações no emocional e social, que uma mudança na dinâmica familiar e de como o idoso se sente dentro do seu contexto podem causar, principalmente em tempos de pandemias, em que uma situação de dependência e redução da capacidade funcional poderá ter grande repercussão na vida das pessoas. ${ }^{18}$

A grande adesão de pessoas idosas com comorbidades às medidas de prevenção na pandemia, pode ter alguma relação com o medo de se infectar e de sofrer prejuízos a saúde ainda maiores. Esse medo pode ter sido gerado por diversas situações: a pandemia além dos graves problemas de abastecimento global, crise econômica, social, ambiental, trabalhista, fiscal e em tantos outros aspectos, gerou também um aprofundamento das vulnerabilidades na vida dos idosos. ${ }^{19} \mathrm{Em}$ uma investigação realizada nos Estados Unidos com 630 adultos com alguma doença crônica, destes $62,7 \%$ eram pessoas idosas com idades entre 60 a 88 anos, o estudo identificou que um em cada quatro participantes (24,6\%) estavam "muito preocupados" em vir a ser infectado pelo novo coronavírus. O medo da ameaça do surto pandêmico foi mais relatado pelas pessoas idosas com 70 anos e 58,6\% informaram que as infecções por coronavírus fez eles mudarem "muito" a sua rotina e atividades de vida diária. ${ }^{20}$

Somado às vulnerabilidades já existentes no processo de envelhecimento; a classificação de grupo de risco no contexto da pandemia; o isolamento social e o maior risco de complicações graves para os idosos com comorbidades, ressalta-se que a escassez de recursos de saúde evidenciada pela pandemia e a necessidade de se fazer escolhas, devido ao aumento de internações em Unidades de Terapia Intensiva (UTI) e o acesso aos respiradores, fizeram com que alguns lugares, a exemplo da Itália, fixassem o critério idade nos protocolos para alocação dos recursos, sem que houvesse muitas reflexões jurídicas, filosóficas e morais, para garantir critérios isonômicos e mitigação de vieses discriminatórios, pode ter gerado uma sensação maior de medo para essa população, corroborada pela omissão do
Estado brasileiro em relação a uma definição clara de como as unidades de saúde deveriam gerenciar os recursos. ${ }^{20}$

Foi observado que pessoas idosas com comorbidades possuem três vezes mais chance de aquisição de informações sobre as infecções por coronavírus, principalmente pela televisão. Destacou-se ainda, estarem sempre e muitas vezes satisfeitos ou até mesmo algumas vezes satisfeitos com as informações que estão sendo veiculadas a respeito do novo coronavírus.

Em outro estudo, os participantes tinham em média cinco doenças crônicas, e média de idade de 59 anos. Os participantes relataram usar a tecnologia com mais frequência para pesquisar informações de saúde (96\%), comunicar-se com profissionais de saúde (92\%), rastrear informações médicas (83\%), rastrear medicamentos $(77 \%)$ e apoiar a tomada de decisão sobre o tratamento (55\%). ${ }^{21}$ Corroborando com esses estudos, participantes idosos de uma pesquisa anterior também informaram a preferência em receber as informações sobre a COVID-19 através de fontes tradicionais, em contrapartida, as pessoas com mais idade e menos instruídas foram identificadas como menos atentos às notícias sobre o novo coronavírus, divergindo dessa investigação. ${ }^{22}$

Dos participantes do presente estudo, 69\% destacaram a televisão como o meio de informação (sempre ou muitas vezes), destaca-se entre os resultados, que $48,15 \%$ utilizam de outros meios de comunicação para aquisição de informações. Esse aspecto se revelou como fator de proteção entre as pessoas idosas com comorbidades quando comparadas com os sem comorbidades.

Embora não tenha sido mencionado o outro meio de comunicação, sabe-se das mudanças no modo de vida das pessoas, ocasionadas pelo avanço tecnológico ocorrido nas últimas décadas. Conhecida como "sociedade da informação", a utilização das ferramentas digitais, como os computadores e celulares é bastante comum. Mesmo que a geração com idade igual ou superior a 60 anos demonstre certo afastamento ou alguma dificuldade de manuseio de tais ferramentas, buscar essa inclusão digital é importante. ${ }^{23}$ Um estudo com 407 pessoas idosas de Israel apontou que houve um aumento no uso de internet pelas pessoas idosas na pandemia da COVID-19, onde os softwares de chat $(61,1 \%)$, recados online $(41,7 \%)$ e jornais online $(40,8 \%)$ foram os mais utilizados no lazer e tarefas online para o enfrentamento do vírus da COVID-19. ${ }^{24}$

A inclusão digital dos idosos estimula o processamento cognitivo, provê informações em tempo real e disponibiliza serviços externos sem a necessidade de se sair de casa, além melhora na velocidade de processamento $(t=3,939 ; p=0,001)$ e na capacidade de planejamento de tarefas $(t=3,504 ; p=0,001) .^{25}$

Um estudo sobre a forma como as informações sobre o novo coronavírus são veiculadas à sociedade, apontou a prevalência de aspectos biológicos da pandemia sob ótica negativa quanto à população idosa, reafirmando preconceitos históricos associados ao envelhecimento, mas, por outro lado, se mostra uma importante ferramenta na divulgação de informações de utilidade pública e de auxílio na situação da pandemia, a exemplo da divulgação 
de reportagens sobre ações sociais de apoio a idosos. Isso evidencia também a necessidade de se reestruturar a forma com que a comunicação é direcionada ao público. ${ }^{25}$

\section{CONCLUSÃO E IMPLICAÇÕES PARA A PRÁTICA}

Conclui-se que os idosos com comorbidades pensam na possibilidade de serem infectados pelo novo coronavírus, concordam mais com as medidas de distanciamento social e se informam mais. Os idosos sem comorbidades, com menores adesões à tais fatores, estão mais susceptíveis e vulnerabilizados diante do cenário pandêmico em curso.

A principal limitação do presente estudo relaciona-se ao delineamento transversal, que não permite o estabelecimento da relação de causa e efeito. Outra limitação foi a não realização de um pré-teste do instrumento com idosos.

Ressalta-se que ainda são escassos os estudos no Brasil que identificam os modos de enfrentamento da pandemia da COVID-19 por pessoas idosas.

O presente estudo aponta para o desafio de ampliar, nesse sentido, a produção de conhecimento da enfermagem, de modo que contribua com a compreensão das especificidades do modo de viver da pessoa idosa diante de medidas como distanciamento social e isolamento. Indica-se a realização de pesquisas com ênfase nos idosos sem comorbidades, para direcionar melhor os cuidados de saúde.

\section{FINANCIAMENTO}

O presente estudo contou com apoio da Fundação de Amparo à Pesquisa do Estado do Amazonas (FAPEAM), Programa de Apoio à Pós-graduação Stricto Sensu (POSGRAD) 2020/2021 - Processo número 062.00702/2020, coordenado por Zilmar Augusto de Souza Filho.

\section{CONTRIBUIÇÕES DOS AUTORES}

Desenho do estudo. Elizabeth Teixeira. Camila Rodrigues Barbosa Nemer.

Coleta ou produção dos dados. Elizabeth Teixeira. Camila Rodrigues Barbosa Nemer. Zilmar Augusto de Souza Filho, André Luiz Machado das Neves, Marcia Helena Machado Nascimento, Bruna Alessandra Costa e Silva Panarra, Paula Andreza Viana Lima.

Análise de dados. Camila Rodrigues Barbosa Nemer. Zilmar Augusto de Souza Filho, André Luiz Machado das Neves, Vanessa Calmont Gusmão Gigante.

Interpretação dos resultados. Camila Rodrigues Barbosa Nemer. Zilmar Augusto de Souza Filho, André Luiz Machado das Neves, Marcia Helena Machado Nascimento, Horacio Pires Medeiros, Paula Andreza Viana Lima, Vera Lúcia Gomes de Oliveira.

Redação e revisão crítica do manuscrito. Zilmar Augusto de Souza Filho, Camila Rodrigues Barbosa Nemer. Elizabeth
Teixeira, André Luiz Machado das Neves, Marcia Helena Machado Nascimento, Horacio Pires Medeiros, Bruna Alessandra Costa e Silva Panarra, Paula Andreza Viana Lima, Vanessa Calmont Gusmão Gigante, Vera Lúcia Gomes de Oliveira.

Aprovação da versão final do artigo. Zilmar Augusto de Souza Filho, Camila Rodrigues Barbosa Nemer. Elizabeth Teixeira, André Luiz Machado das Neves, Marcia Helena Machado Nascimento, Horacio Pires Medeiros, Bruna Alessandra Costa e Silva Panarra, Vanessa Calmont Gusmão Gigante, Vera Lúcia Gomes de Oliveira.

Responsabilidade por todos os aspectos do conteúdo e a integridade do artigo publicado. Zilmar Augusto de Souza Filho, Camila Rodrigues Barbosa Nemer. Elizabeth Teixeira, André Luiz Machado das Neves, Marcia Helena Machado Nascimento, Horacio Pires Medeiros, Bruna Alessandra Costa e Silva Panarra, Paula Andreza Viana Lima, Vanessa Calmont Gusmão Gigante, Vera Lúcia Gomes de Oliveira.

\section{EDITOR ASSOCIADO}

\author{
Gerson Luiz Marinho
}

\section{REFERÊNCIAS}

1. Hui DS, Azhar E, Madani TA, Ntoumi F, Kock R, Dar O et al. The continuing 2019-nCoV epidemic threat of novel coronaviruses to global health - The latest 2019 novel coronavirus outbreak in Wuhan, China. Int J Infect Dis. 2020;91:264-6. http://dx.doi.org/10.1016/j.ijid.2020.01.009. PMid:31953166.

2. World Health Organization. Coronavirus disease 2019 (COVID-19) Situation Report - 51. Geneva: WHO; 2020.

3. Yang J, Zheng Y, Gou X, Pu K, Chen Z, Guo Q et al. Prevalence of comorbidities in the novel Wuhan coronavirus (COVID-19) infection: A systematic review and meta-analysis. Int J Infect Dis. 2020;2020(94):915. http://dx.doi.org/10.1016/j.jijid.2020.03.017. PMid:32173574.

4. Liu K, Chen Y, Lin R, Han K. Clinical features of COVID-19 in elderly patients: A comparison with young and middle-aged patients. J Infect. 2020;80(6):E14-8. http://dx.doi.org/10.1016/j.jinf.2020.03.005. PMid:32171866.

5. Centers for Disease Control and Prevention. National Center for Health Statistics (NCHS). Weekly Updates by select demographic and geographic characteristics. Provisional Death Counts for Coronavirus Disease 2019 (COVID-19). [Internet] CDC; 2021 [citado 2021 jan 27]. Disponível em: https://www.cdc.gov/nchs/nvss/vsrr/covid_weekly/index. $\mathrm{htm}$

6. Centers for Disease Control and Prevention. Increased Risk of Hospitalization or Death Age Increases Risk for Severe Illness. [Internet] CDC; 2021 [citado 2021 jan 27]. Disponível em https://www.cdc.gov/ coronavirus/2019-ncov/need-extra-precautions/older-adults.html.

7. Ministério da Saúde (BR). Secretaria de Vigilância em Saúde. Boletim epidemiológico especial - Doença pelo Coronavírus COVID-19. Semana Epidemiológica 52 (20/12 a 26/12). [Internet] 2020 [citado 2021 jan 23]. Disponível em: https://www.gov.br/saude/pt-br/media/pdf/2020/ dezembro/30/boletim_epidemiologico_covid_43_final_coe.pdf

8. Associação Brasileira de Saúde Coletiva. Bolsonaro, inimigo da saúde do povo - Nota das entidades da saúde coletiva e da bioética a respeito do pronunciamento do Presidente da República em cadeia nacional de rádio e TV, em 24 de março. [Internet]. [citado 2020 mar 25]. Disponível em: https://abrasco.org.br/hotsites/nota-covid19/

9. De Troi M, Quintilio W. Coronavírus: lições anti-negacionistas e o futuro do planeta. SciELO em Perspectiva [Internet]. 2020 [citado 2020 jun 25]. Disponível em: https://blog.scielo.org/blog/2020/03/31/coronaviruslicoes-anti-negacionistas-e-o-futuro-do-planeta/ 
10. Pereira DS, Nogueira JAD, Silva CAB. Qualidade de vida e situação de saúde de idosos: um estudo de base populacional no Sertão Central do Ceará. Rev Bras Geriatr Gerontol. 2015;18(4):893-908. http://dx.doi. org/10.1590/1809-9823.2015.14123.

11. Confortin SC, Schneider IJC, Antes DL, Cembranel F, Ono LM, Marques LP et al. Condições de vida e saúde de idosos: resultados do estudo de coorte EpiFloripa Idoso. Epidemiol Serv Saude. 2017 jun;26(2):305-17. http://dx.doi.org/10.5123/S1679-49742017000200008. PMid:28492772.

12. Almeida JS, Cardoso JÁ, Cordeiro EC, Lemos M, Araújo TME, Sardinha AHL. Caracterização epidemiológica dos casos de covid-19 no maranhão: Uma breve análise. [Internet]. 2020 [citado 2020 set 2020]. Disponível em: https://preprints.scielo.org/index.php/scielo/preprint/view/314/377

13. Ruan Q, Yang K, Wang W, Jiang L, Song J. Clinical predictors of mortality due to COVID-19 based on an analysis of data of 150 patients from Wuhan, China. Intensive Care Med. 2020;46(5):846-8. http://dx.doi. org/10.1007/s00134-020-05991-x. PMid:32125452.

14. Banerjee D. 'Age and ageism in COVID-19': Elderly mental health-care vulnerabilities and needs. Asian J Psychiatr. [Internet]. 2020 jun [citado 2020 set 14;51:02154. Disponível em: https://pubmed.ncbi.nlm.nih gov/32403024/

15. Santini ZI, Jose PE, York Cornwell E, Koyanagi A, Nielsen L, Hinrichsen $\mathrm{C}$ et al. Social disconnectedness, perceived isolation, and symptoms of depression and anxiety among older Americans (NSHAP): a longitudinal mediation analysis. Lancet Public Health. 2020 jan;5(1):e62-70. http:// dx.doi.org/10.1016/S2468-2667(19)30230-0. PMid:31910981 .

16. Rocha ACAL, Ciosak SI. Chronic Disease in the elderly: spirituality and coping. Rev Esc Enferm USP. 2014;48(spe2):87-93. https://doi. org/10.1590/S0080-623420140000800014

17. Meng H, XuY, Jiali X, Zhang Y, Liu B, Yang H. Analyze the psychological impact of COVID-19 among the elderly population in China and make corresponding suggestions. Psychiatry Res. 2020 jun;289:112983. http://dx.doi.org/10.1016/j.psychres.2020.112983.
18. Oliveira LPBA, Menezes RMP. Representações de fragilidade para idosos no contexto da estratégia saúde da família. Texto Contexto Enferm. 2011;20(2):301-9. http://dx.doi.org/10.1590/S0104-07072011000200012.

19. Dadalto L, Mascarenhas II, Matos ACH. Salvem também os idosos: etarismo e a alocação de recursos na realidade brasileira de combate à COVID. civilistica.com [Internet]. 2020 ago [citado 2020 set 14];9(2):119. Disponível em: http://civilistica.com/wp-content/uploads1/2020/08/ Dadalto-Mascarenhas-e-Matos-civilistica.com-a.9.n.2.2020-1.pdf

20. Wolf MS, Serper M, Opsasnick L, O'Conor RM, Curtis L, Benavente JY et al. Awareness, attitudes, and actions related to COVID-19 among adults with chronic conditions at the onset of the U.S outbreak: a crosssectional survey. Ann Intern Med. 2020;173(2):100-9. http://dx.doi org/10.7326/M20-1239. PMid:32271861.

21. Zulman DM, Jenchura EC, Cohen DM, Lewis ET, Houston TK, Asch SM How can ehealth technology address challenges related to multimorbidity? Perspectives from patients with multiple chronic conditions. J Gen Intern Med. 2015;30(8):1063-70. http://dx.doi.org/10.1007/s11606-015-32229. PMid:25691239.

22. Chen X, Gao H, Zou Y, Lin F. Changes in psychological wellbeing, atititude, and information-seeking behavior among people at the epicenter of the COVID-19 pandemic: a panel survey of residentes in Hubei province, China. Epidemiol Infect. 2020;148(e201):1-10. http:// dx.doi.org/10.1017/S0950268820002009. PMid:32873358.

23. Banhato EFC, Silva KCA, Magalhães NC, Mota ME, Guedes DV, Scoralick NN. Inclusão digital: ferramenta de promoção para envelhecimento cognitivo, social e emocional saudável? Psicol Hosp. 2020;5(2):2-20.

24. Nimrod G. Changes in internet use when coping stress: older adults during the COVID-19 pandemic. Am J Geriatr Psychiatry. 2020 out;28(10):10204. http://dx.doi.org/10.1016/j.jagp.2020.07.010. PMid:32771312.

25. Leão LRB, Ferreira VHS, Faustino AM. O idoso e a pandemia do Covid-19: uma análise de artigos publicados em jornais. Braz J Develop. 2020;6(7):45123-42. http://dx.doi.org/10.34117/bjdv6n7-218. 